\title{
THE STRONG SYMMETRIC GENUS OF THE HYPEROCTAHEDRAL GROUPS
}

\author{
MICHAEL A. JACKSON
}

\section{INTRODUCTION}

In the study of Reimannian manifolds, it is natural to consider the finite groups, which act as automorphisms of the manifold. On the other hand, given a finite group, one may consider the topological surfaces on which the group acts faithfully as a group of automorphisms. There are several natural invariants assigned to a group that are associated to the action of that group on compact orientable surfaces. The first of these invariants is the genus $\gamma(G)$ of the group $G$, which is the smallest genus of a surface on which some Cayley graph for $G$ can be embedded. The symmetric genus $\sigma(G)$ of a finite group $G$ is the smallest genus of a surface on which $G$ acts faithfully as a group of automorphisms. The strong symmetric genus $\sigma^{0}(G)$ is the smallest genus of a surface on which $G$ acts faithfully as a group of orientation preserving automorphisms. (See [11] chapter 6.) It is clear that these invariants satisfy the inequality $\gamma(G) \leq \sigma(G) \leq \sigma^{0}(G)$.

Perhaps the most classical of these parameters is the strong symmetric genus. It was first considered by Burnside [2, who included the further restriction that the quotient space under the action be a sphere. The strong symmetric genus is what we will concern ourselves with in this paper. Various results have been shown involving the strong symmetric genus. For example, all groups $G$ such that $\sigma^{0}(G) \leq 3$ have been computed (see [1, 12]). Also the strong symmetric genus of several infinite families of finite groups have been found, such as the alternating and symmetric groups [3, 4, 5, the groups $P S L_{2}(q)$ [9, 10, and the groups $S L_{2}(q)$ [18]. In addition, the strong symmetric genus has been found for the sporadic finite simple groups [6, 21, 22, 23].

Since the strong symmetric genus of the alternating and symmetric groups is known, it is natural to try to find the strong symmetric genus for other infinite families of finite groups. An example of such an infinite family is the collection of hyperoctahedral groups. Recall that the hyperoctahedral groups are the finite Coxeter groups of type $B_{n}$. The hyperoctahedral group $B_{n}$ for $n \geq 3$ is defined as the group of symmetries of the $n$-dimensional cube. In this paper we will prove the following theorem and a corollary about the hyperoctahedral groups:

Theorem 1. For all $n \geq 3$, except $n=5,6$, and 8 , the hyperoctahedral group $B_{n}$ is a quotient of the triangle group $T(2,4,6)=\left\langle x, y, z \mid x^{2}=y^{4}=z^{6}=x y z=1\right\rangle$. In 
the exceptional cases, $B_{5}, B_{6}$ and $B_{8}$ are quotients of the triangle groups $T(2,4,10)$, $T(2,6,6)$, and $T(2,4,8)$ respectively.

Corollary 2. For all $n \geq 3$, except $n=5,6$, and 8, the strong symmetric genus of the hyperoctahedral group $B_{n}$ is $\frac{n ! 2^{n}}{24}+1=\frac{n ! 2^{n-3}}{3}+1$. The strong symmetric genus in the exceptional cases $B_{5}, B_{6}$, and $B_{8}$ are 289, 3841, and 645,121 respectively.

The investigation of the strong symmetric genus of the hyperoctahedral groups grew out of a VIGRE Working Group at The Ohio State University during the fall of 2002. In this working group, we were looking to find the strong symmetric genus of some finite Coxeter groups. The exceptional cases of Theorem 1 were found by the working group [17].

\section{Subgroups of the Hyperoctahedral groups}

For the present time we will fix an $n \geq 3$ and look at the hyperoctahedral group $B_{n}$. As we have previously said, $B_{n}$ is defined as the group of symmetries of the $n$-dimensional cube. This group is also described as the wreath product $\mathbb{Z}_{2} \prec \Sigma_{n}$. Another description of this group is as the group of all $n \times n$ signed permutation matrices. For our purposes, we will use the wreath product description as adopted by V. S. Sikora [13]. For an element of $B_{n}$, we will write a tuple $[\sigma, b]$ where $\sigma$ is an element of $\Sigma_{n}$ and $b$ is a list of $n$ binary digits representing the element of $\left(\mathbb{Z}_{2}\right)^{n}$. The multiplication then becomes $[\sigma, b] \cdot[\tau, c]=\left[\sigma \cdot \tau, \tau^{-1}(b)+c\right]$ where addition in the binary digits is a parity computation. We will use the convention of calling $b$ even or odd according to the number of ones appearing as binary digits of $b$. Notice that if $b$ and $c$ have the same parity, then $b+c$ is even, and if they differ in parity, then $b+c$ is odd.

Recall that the following sequence is a split exact sequence of groups:

$$
\left(\mathbb{Z}_{2}\right)^{n} \stackrel{i}{\rightarrow} B_{n} \stackrel{\pi}{\rightarrow} \Sigma_{n}
$$

where $i(b)=[1, b]$ and $\pi([\sigma, b])=\sigma$. We will also assume that $s: \Sigma_{n} \rightarrow B_{n}$ with $\pi \circ s=i d_{\Sigma_{n}}$. Proper subgroups $G \subset B_{n}$ such that $\pi(G)=\Sigma_{n}$ are of particular interest here. Specifically we wish to examine maximal subgroups of $B_{n}$ with this property. Throughout this discussion, we will assume that $n \geq 5$. In order to examine the maximal subgroups of $B_{n}$ with $\pi(G)=\Sigma_{n}$, we need to introduce another maximal subgroup of $B_{n}$. Let $C_{n}$ be the subgroup of $B_{n}$ consisting of all elements $[\sigma, b]$ such that $\sigma \in A_{n}$. It is immediately clear that $\left[B_{n}: C_{n}\right]=2$ and $C_{n}=\mathbb{Z}_{2} \prec A_{n}$. So we get the following commutative diagram

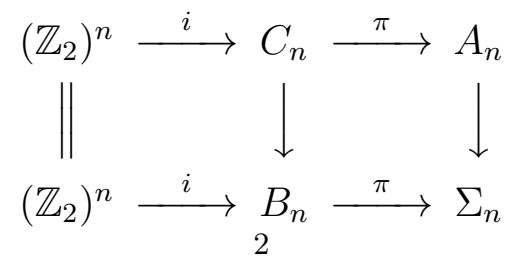


where both horizontal sequences are split exact.

Let $G \subset B_{n}$ be a subgroup such that $\pi(G)=\Sigma_{n}$. Let $H=G \cap C_{n}$. We, thus, see that $\pi(H)=A_{n}$ and $[G: H]=2$. Letting $K=\operatorname{ker}\left(\left.\pi\right|_{H}\right)$ gives the following commutative diagram:

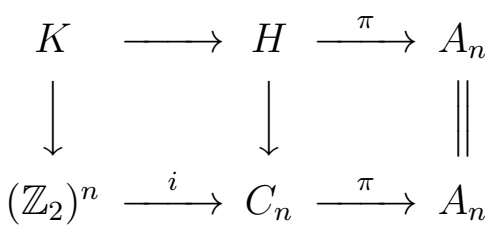

Notice that $C_{n}$ acts on $\left(\mathbb{Z}_{2}\right)^{n}$ by conjugation with kernel $i\left(\left(\mathbb{Z}_{2}\right)^{n}\right)$. Since $K \triangleleft H$, we see that $K$ must be invariant under the action of $A_{n}$. Now we will look at the various possibilities for $K$ and the implications for $H$.

The first and most obvious case is that $K \cong\left(\mathbb{Z}_{2}\right)^{n}$ and $H=C_{n}$. In the other extreme lies the case where $K=1, H \cong A_{n}$. The third straightforward case is that $K=Z\left(C_{n}\right)$ and so $H \cong \mathbb{Z}_{2} \times A_{n}$. The fourth case is the most interesting one for this discussion and occurs when $K \cong\left(\mathbb{Z}_{2}\right)^{n-1}$ where each generator of $K$ is mapped to the product of two generators in $\left(\mathbb{Z}_{2}\right)^{n} \subset C_{n}$. Notice that $\left[C_{n}: H\right]=2$, so $H \triangleleft C_{n}$. Let $s$ be any splitting homomorphism of the split exact sequence defining $C_{n}$. Then $s\left(A_{n}\right) \cap H \triangleleft s\left(A_{n}\right)$ and $\left[s\left(A_{n}\right): H \cap s\left(A_{n}\right)\right] \leq 2$. Since $n \geq 5, A_{n}$ is simple, so $s\left(A_{n}\right) \subset H$. By realizing that these are the only possible cases for $K$, we have led ourselves to the following proposition:

Proposition 3. For $n \geq 5$, let $H$ be a subgroup of $C_{n}$ with $\pi(H)=A_{n}$; then $H$ is a split extension of $A_{n}$ by one of the following: $1, \mathbb{Z}_{2},\left(\mathbb{Z}_{2}\right)^{n-1}$, or $\left(\mathbb{Z}_{2}\right)^{n}$. In the first two cases, $H$ is isomorphic to $A_{n}$ and $\mathbb{Z}_{2} \times A_{n}$ respectively. In the last case, $H=C_{n}$, and in the third case $H=\left\{[\sigma, b] \in C_{n} \mid b\right.$ is even $\}$.

We now use the following commutative diagram to relate Proposition 3 to the group $G$ :

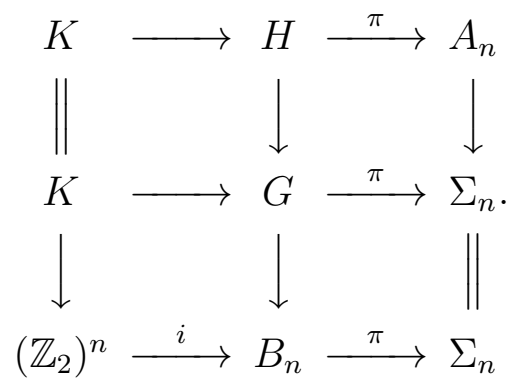

Straightforward calculations using the fact that $[G: H]=2$ give us the following proposition:

Proposition 4. For $n \geq 5$, let $G$ be a subgroup of $B_{n}$ with $\pi(G)=\Sigma_{n}$; then $G$ is a split extension of $\Sigma_{n}$ by one of the following: $1, \mathbb{Z}_{2},\left(\mathbb{Z}_{2}\right)^{n-1}$, or $\left(\mathbb{Z}_{2}\right)^{n}$. In the first two cases, $G$ is isomorphic to $\Sigma_{n}$ and $\mathbb{Z}_{2} \times \Sigma_{n}$ respectively. In the last case $G=B_{n}$, 
and in the third case either

$$
G=\left\{[\sigma, b] \in B_{n} \mid b \text { is even }\right\} \text { or } G=\left\{[\sigma, b] \in B_{n} \mid \begin{array}{c}
b \text { is even if } \sigma \in A_{n} \\
b \text { is odd if } \sigma \in \Sigma_{n} \backslash A_{n}
\end{array}\right\} .
$$

\section{Generators in $B_{n}$}

In order to find the strong symmetric genus of a group, it will be important to find a pair of generators. Of particular interest will be the orders of the generators in this pair as well as the order of their product. In the following proposition, we will show that in the case of the hyperoctahedral groups, these orders will all be even.

Proposition 5. If $[\sigma, b]$ and $[\tau, c]$ generate $B_{n}$, then the orders of $[\sigma, b],[\tau, c]$, and their product $\left[\sigma \cdot \tau, \tau^{-1}(b)+c\right]$ all are even.

Proof: Suppose that one of the orders is odd. Without loss of generality, we may assume that $[\sigma, b]$ has odd order. Clearly $\sigma$ and $\tau$ must generate $\Sigma_{n}$. Since $[\sigma, b]$ has odd order, $\sigma$ must also have odd order; therefore, $\sigma \in A_{n}, \tau \in \Sigma_{n} \backslash A_{n}$, and $\sigma \cdot \tau \in \Sigma_{n} \backslash A_{n}$. So we see that $\tau$ and $\sigma \cdot \tau$ have even orders.

Let $k$ be the order of $\sigma$. Since $[\sigma, b]$ has odd order, $[\sigma, b]^{k}=1$. Notice that $b$ must be even since $[\sigma, b]^{k}=\left[1, \sigma^{-k+1}(b)+\sigma^{-k+2}(b)+\cdots+\sigma^{-1}(b)+b\right]$ and a sum of an odd number of odd elements is odd. If $c$ is also even, then $[\sigma, b]$ and $[\tau, c]$ both lie in the proper subgroup $\left\{[\sigma, b] \in B_{n} \mid b\right.$ is even $\} \subset B_{n}$ and, therefore, cannot generate $B_{n}$. If on the other hand $c$ is odd, then $[\sigma, b]$ and $[\tau, c]$ both lie in the proper subgroup

$$
\left\{\begin{array}{l|l}
{[\sigma, b] \in B_{n} \mid \begin{array}{c}
b \text { is even if } \sigma \in A_{n} \\
b \text { is odd if } \sigma \in \Sigma_{n} \backslash A_{n}
\end{array}}
\end{array}\right\} \subset B_{n}
$$

and, therefore, cannot generate $B_{n}$. Thus the order of $[\sigma, b]$ must also be even.

Notice that if $[\sigma, b]$ and $[\tau, c]$ generate $B_{n}$, then $\sigma$ and $\tau$ must generate $\Sigma_{n}$. Since we are looking to find generators of $B_{n}$, we need to first find generators of $\Sigma_{n}$. The second step in the process is to construct generators of $B_{n}$ from the generators of $\Sigma_{n}$. Also during this construction we would like to have control of the orders of the generators of $B_{n}$ as well as of their product. The following proposition gives the results of the process we will use to construct generators of $B_{n}$ from those of $\Sigma_{n}$.

Proposition 6. Suppose $\sigma$ and $\tau$ generate $\Sigma_{n}$ as $\operatorname{Symm}(\Gamma)$ where $\Gamma=\{1,2, \ldots, n\}$ such that $\sigma \cdot \tau \in \Sigma_{n} \backslash A_{n}$ where $\sigma, \tau$, and $\sigma \cdot \tau$ all have even order. Futhermore, assume that $\sigma$ fixes an element $i \in \Gamma$ and $\tau$ fixes at least three elements of $\Gamma$, one of which we call $j$ such that $i$ and $j$ are in the same cycle of the element $\sigma \cdot \tau$. Let $b=$ $(0, \ldots, 0,1,0, \ldots, 0)$ where the 1 is in the $i^{\text {th }}$ position, and let $c=(0, \ldots, 0,1,0, \ldots, 0)$ where the 1 is in the $j^{\text {th }}$ position. Under these conditions, $[\sigma, b]$ and $[\tau, c]$ generate $B_{n}$. In addition the elements $[\sigma, b],[\tau, c]$ and $\left[\sigma \cdot \tau, \tau^{-1}(b)+c\right]$ have the same orders as $\sigma, \tau$, and $\sigma \cdot \tau$ respectively.

Proof: The results about the orders of $[\sigma, b]$ and $[\tau, c]$ are obvious since $\sigma(b)=b$ and $\tau(c)=c$. On the other hand, $(\sigma \cdot \tau)^{-k}\left(\tau^{-1}(b)+c\right)=(\sigma \cdot \tau)^{-k-1}(b)+(\sigma \cdot \tau)^{-k}(c)$. 
Notice that if $k$ is the length of the cycle in $\sigma \cdot \tau$ that contains $i$ and $j$, then $[\sigma$. $\left.\tau, \tau^{-1}(b)+c\right]^{k}=\left[(\sigma \cdot \tau)^{k},(0,0, \ldots, 0,0)\right]$ since the images of $b$ will put a 1 in each position corresponding to the elements in the cycle and the images of $c$ will put a 1 in each position corresponding to the elements in the same cycle. Thus it is clear that the order of $\left[\sigma \cdot \tau, \tau^{-1}(b)+c\right]$ is the same as the order of $\sigma \cdot \tau$.

Let $G=\langle[\sigma, b],[\tau, c]\rangle \subset B_{n}$. We need to show that $G=B_{n}$. Notice that $G$ is a subgroup of $B_{n}$ such that $\pi(G)=\Sigma_{n}$. So from Proposition 4 we know that $G$ is a split extension of $\Sigma_{n}$ by one of the following: $1, \mathbb{Z}_{2},\left(\mathbb{Z}_{2}\right)^{n-1}$, or $\left(\mathbb{Z}_{2}\right)^{n}$.

Recall that any section $s: \Sigma_{n} \rightarrow B_{n}$ takes $\alpha \in A_{n}$ to $[1, d] \cdot[\alpha,(0, \ldots, 0)] \cdot[1, d]^{-1}$ for some $[1, d] \in B_{n}$, and if $\alpha \in \Sigma_{n} \backslash A_{n}$, then either $s(\alpha)=[1, d] \cdot[\alpha,(0, \ldots, 0)] \cdot[1, d]^{-1}$ or $s(\alpha)=[1, d] \cdot[\alpha,(1, \ldots, 1)] \cdot[1, d]^{-1}$. Notice if $\alpha \in A_{n}, s(\alpha)=[\alpha, a]$ where $a$ is even.

Suppose first that $G$ is a split extension of $\Sigma_{n}$ by 1 . Then $[\sigma, b]$ and $[\tau, c]$ must be in the image of some section homorphism $s: \Sigma_{n} \rightarrow B_{n}$. Either $\sigma \in A_{n}$ or $\tau \in A_{n}$, however, and both $b$ and $c$ are odd. So $G$ is not a split extension of $\Sigma_{n}$ by 1 .

Suppose that $G$ is a split extension of $\Sigma_{n}$ by $\mathbb{Z}_{2}$. This implies that for some section homorphism, $s: \Sigma_{n} \rightarrow B_{n}, G=Z\left(B_{n}\right) \times s\left(\Sigma_{n}\right)$. If $n$ is even, then the only elements in $Z\left(B_{n}\right) \times s\left(\Sigma_{n}\right)$ of the form $[\alpha, a]$ with $\alpha \in A_{n}$ have an even $a$. Clearly either $[\sigma, b] \notin Z\left(B_{n}\right) \times s\left(\Sigma_{n}\right)$ or $[\tau, c] \notin Z\left(B_{n}\right) \times s\left(\Sigma_{n}\right)$; therefore, we may assume that $n$ is odd. Suppose that $\tau \in A_{n}$. So $[\tau, c]=[1,(1, \ldots, 1)] \cdot[1, d] \cdot[\tau,(0, \ldots, 0)] \cdot[1, d]^{-1}$ for some $[1, d] \in B_{n}$. It follows that $c=(1, \ldots, 1)+\tau^{-1}(d)+d$, which cannot be the case since $\tau$ fixes an element besides $j$. So we may also assume that $\tau \in \Sigma_{n} \backslash A_{n}$.

Since $\tau \in \Sigma_{n} \backslash A_{n}$, either $[\tau, c]=[1,(1, \ldots, 1)] \cdot[1, d] \cdot[\tau,(0, \ldots, 0)] \cdot[1, d]^{-1}$ or $[\tau, c]=[1, d] \cdot[\tau,(1, \ldots, 1)] \cdot[1, d]^{-1}$ for some $[1, d] \in B_{n}$. In either case, $c=$ $(1, \ldots, 1)+\tau^{-1}(d)+d$, which as before cannot be the case since $\tau$ fixes an element besides $j$. So $G$ is not a split extension of $\Sigma_{n}$ by $\mathbb{Z}_{2}$.

Suppose that $G$ is a split extension of $\Sigma_{n}$ by $\left(\mathbb{Z}_{2}\right)^{n-1}$. By Proposition 4 , either

$$
G=\left\{[\sigma, b] \in B_{n} \mid b \text { is even }\right\} \text { or } G=\left\{\begin{array}{l|l}
{[\sigma, b] \in B_{n}} & \begin{array}{c}
b \text { is even if } \sigma \in A_{n} \\
b \text { is odd if } \sigma \in \Sigma_{n} \backslash A_{n}
\end{array}
\end{array}\right\} .
$$

Recall that either $\sigma \in A_{n}$ or $\tau \in A_{n}$. If $\sigma \in A_{n}$, then $[\sigma, b] \notin G$. If $\tau \in A_{n}$, then $[\tau, c] \notin G$. So $G$ is not a split extension of $\Sigma_{n}$ by $\left(\mathbb{Z}_{2}\right)^{n-1}$.

The only possibility left is that $G$ is a split extension of $\Sigma_{n}$ by $\left(\mathbb{Z}_{2}\right)^{n}$. Thus we see that $G=B_{n}$ and so the proposition is proven.

\section{GENERATING PAIRS}

If a finite group $G$ has generators $x$ and $y$ of orders $p$ and $q$ respectively with $x y$ having the order $r$, then we say that $(x, y)$ is a $(p, q, r)$ generating pair of $G$. By the obvious symmetries concerning generators, we will use the convention that $p \leq q \leq r$.

Following the convention of Marston Conder in [5], we say that a $(p, q, r)$ generating 
pair of $G$ is a minimal generating pair if there does not exist a $(k, l, m)$ generating pair for $G$ with $\frac{1}{k}+\frac{1}{l}+\frac{1}{m}>\frac{1}{p}+\frac{1}{q}+\frac{1}{r}$.

Recall that the groups of small strong symmetric genus are well known (see [1, 12). So for now we will assume that $\sigma^{0}(G)>1$. The hyperoctahedral groups have strong symmetric genus at least 2. It is also known that for groups with $\sigma^{0}(G)>1$, any generating pair will be a $(p, q, r)$ generating pair with $\frac{1}{p}+\frac{1}{q}+\frac{1}{r}<1$. Using the Riemann-Hurwitz equation, we see that given any generating pair of $G$, we get an upper bound on the strong symmetric genus of $G$ [15]. If $G$ has a $(p, q, r)$ generating pair, then

$$
\sigma^{0}(G) \leq 1+\frac{1}{2}|G| \cdot\left(1-\frac{1}{p}-\frac{1}{q}-\frac{1}{r}\right)
$$

The following lemma, which is a result of Singerman [14] (see also [12, 16]), shows that the strong symmetric genus for most groups is computed directly from a minimal generating pair.

Lemma 7 (Singerman [14]). Let $G$ be a finite group such that $\sigma^{0}(G) \geq 2$. If $|G|>$ $12\left(\sigma^{0}(G)-1\right)$, then $G$ has a $(p, q, r)$ generating pair with

$$
\sigma^{0}(G)=1+\frac{1}{2}|G| \cdot\left(1-\frac{1}{p}-\frac{1}{q}-\frac{1}{r}\right) .
$$

In addition, we may assume that either $p=2$, or $p=q=3$ and $r$ is 4 or 5 .

Applying Lemma 7 to our situation with the hyperoctahedral groups results in the following corollary.

Corollary 8. Let $n \geq 3$ and $G=B_{n}$. If $|G|>12\left(\sigma^{0}(G)-1\right)$, then $G$ has a $(p, q, r)$ generating pair with

$$
\sigma^{0}(G)=1+\frac{1}{2}|G| \cdot\left(1-\frac{1}{p}-\frac{1}{q}-\frac{1}{r}\right) .
$$

In addition, we may assume that $p=2, q=4$, and $r$ is either 6,8 or 10 .

Proof: Since all groups $H$ with $\sigma^{0}(H)<2$ have been classified, we know that $\sigma^{0}(G) \geq 2$. We also know that if $G$ has a $(p, q, r)$ generating pair, then $p, q$, and $r$ must all be even. The rest of the corollary follows from straightforward calculations and by recalling that $|G|=n ! 2^{n}$.

In light of Corollary 8 . Corollary 2 is a direct result of Theorem 1, We will see that the exceptional cases of Theorem 1 will be shown by using GAP [8]. For the remaining cases, we will show the existence of a $(2,4,6)$ generating pair for $B_{n}$ by constructing it from a $(2,4,6)$ generating pair for $\Sigma_{n}$ using Proposition 6 .

\section{SPECIAL GENERATORS OF $\Sigma_{n}$}

First we will need to recall a theorem of Jordan (see [19]). 
Theorem 9 (Jordan [19]). Let $G$ be a transitive permutation group on a set of size $n$ such that $G$ is primitive and contains a p-cycle for some prime $p<n-2$. Then $G$ is either $A_{n}$ or $\Sigma_{n}$.

We will use this theorem by applying the following corollary. The method that we will use follows that of Marston Conder (see [3, 4]).

Corollary 10. Let $G=\langle\sigma, \tau\rangle$ be a transitive permutation group on a set $\Gamma$ of size $n$. Assume that for some prime $p<n-2, G$ contains a $p$-cycle $z$ such that the points permuted by $z$ include an element $a \in \Gamma$ as well as its image under $\sigma$ and an element $b \in \Gamma$ as well as its image under $\tau$. Then $G$ is either $A_{n}$ or $\Sigma_{n}$.

Proof: Using Theorem 9, the only part we need to prove is that $G$ is a primitive permutation group on $\Gamma$. Suppose that $G$ acts imprimitively on $\Gamma$. Let $z$ be the $p$ cycle in the hypothesis. Since $z$ fixes all the other points of $\Gamma$, the $p$ points permuted by $z$ must lie in the same block of imprimitivity $B$. By hypothesis, however, $a$ and $\sigma(a)$ are both in $B$; therefore, $\sigma$ fixes $B$. Also by hypothesis, $b$ and $\tau(b)$ are both in $B$; therefore, $\tau$ fixes $B$. This implies that the block $B$ is fixed by all of $G$, contradicting the transitivity of $G$.

At this point we have done all of the preliminary work; it is now time to prove the main theorem. To begin we point out that the result for the groups $B_{n}$ with $3 \leq n \leq 40$ were shown using GAP [8]. Some of these results were shown in [17. For the groups $B_{n}$ with $n \geq 41$, we show that each group $\Sigma_{n}$ with $n \geq 41$ can be generated by two elements $\sigma$ and $\tau$. Using Corollary [10, this process will involve finding two elements $\sigma$ and $\tau$ such that these two elements generate a transitive permutation group on $n$ letters such that some element of $\langle\sigma, \tau\rangle$ is a $p$-cycle, which contains a letter and its image under $\sigma$ and a possibly different letter and its image under $\tau$. In order to be able to construct a $(2,4,6)$ generating pair, $([\sigma, b],[\tau, c])$, of $B_{n}$ from $\sigma$ and $\tau$ as in Proposition [6 we will require that the following hold:

(1) $\sigma$ has order 4 and $\tau$ has order 2,

(2) $\sigma$ fixes a letter $i$,

(3) $\tau$ fixes at least three letters one of which we call $j$,

(4) $\sigma \cdot \tau \in \Sigma_{n} \backslash A_{n}$,

(5) $i$ and $j$ are in the same cycle of the element $\sigma \cdot \tau$,

(6) $\langle\sigma, \tau\rangle$ is a transitive permutation group on $n$ letters, and

(7) some element of $\langle\sigma, \tau\rangle$ is a $p$-cycle, which contains a letter and its image under $\sigma$ and a possibly different letter and its image under $\tau$.

\section{Coset Diagrams and Results}

We will demonstrate the existence of the desired elements $\sigma$ and $\tau$ in each $\Sigma_{n}$ with $n \geq 41$ by using diagrams which are called coset diagrams. First let us give a brief introduction to these diagrams by looking at an example in Figure 1. The diagram in Figure 1 is a representation of the group $\Sigma_{19}$. The diagram gives two elements 


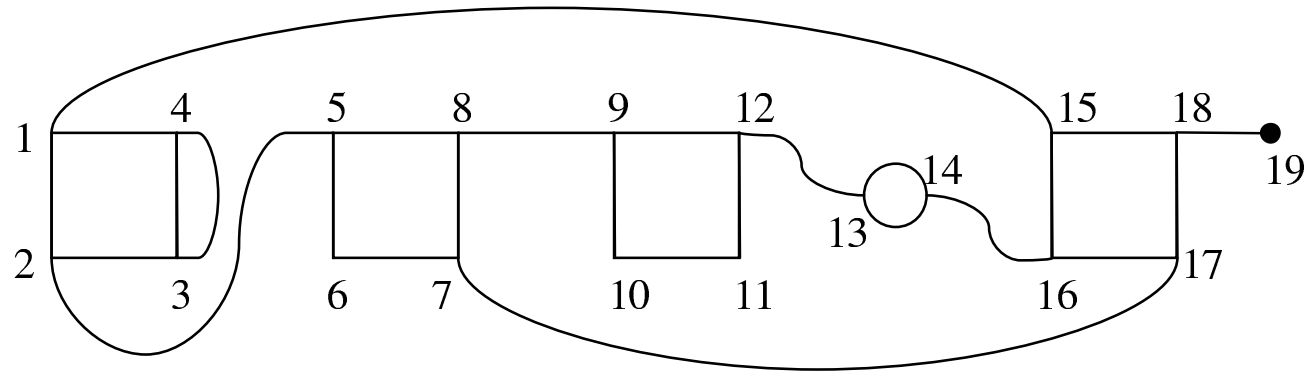

Figure 1. Example $\Sigma_{19}$

of a transitive permutation group on 19 letters. The first element $\sigma$ is given by the squares and circles in the diagram and will have order 4. A cycle of $\sigma$ is given by reading the labels in a counter-clockwise direction. So in this case,

$$
\sigma=\left(\begin{array}{lll}
1 & 3 & 4
\end{array}\right)(5678)(91011 \text { 12)(13 14)(15 } 1617 \text { 18). }
$$

The second element $\tau$ is given by the other lines and curves in the diagram. $\tau$ will be an involution with a 2-cycle, for each line or curve, that is the cycle of its two labels. In this case we see

$$
\tau=(1 \text { 15)(2 5)(3 4)(7 17)(8 9)(12 13)(14 16)(18 19). }
$$

To find to where an integer is mapped under the element $\sigma \cdot \tau$, we start at that label, trace along a square or circle in the counter-clockwise direction if possible, and then trace a curve not in one of these shapes if possible. The permutation given by a general word in $\sigma$ and $\tau$ can be found in a similar way.

We see that the element $\sigma^{2} \cdot \tau$ has four 4-cycles and one 3-cycle, which is (10 1312 ). By Corollary 10, we see that $\sigma$ and $\tau$ generate the symmetric group on 19 elements. Using the elements $\sigma$ and $\tau$ and creating elements $[\sigma, b]$ and $[\tau, c]$ of $B_{19}$ as in Proposition [6] we also see that $[\sigma, b]$ and $[\tau, c]$ generate $B_{19}$.

In a similar way as the example above, we will now look at Figure 2, which gives a coset digram for the group $\Sigma_{41}$. As with the example in Figure 1, these figures show that the group $B_{41}$ is a $(2,4,6)$ group. The necessary information about $\langle\sigma, \tau\rangle$ containing a $p$-cycle, which contains a letter and its image under $\sigma$ and a possibly different letter and its image under $\tau$, can be found in Table I. Similar coset diagrams exist for the groups $\Sigma_{n}$ with $42 \leq n \leq 52$ and the corresponding information is also contained in Table I. These diagrams show that each group $B_{n}$ for $42 \leq n \leq 52$ is a $(2,4,6)$ groups.

To show the result that the groups $B_{n}$ with $n>52$ are all $(2,4,6)$ groups, we will recursively add the piece shown in Figure 3 . We attach the piece by matching up the points $a$ and $b$ and then making the $a^{\prime}$ and $b^{\prime}$ into a new $a$ and $b$ respectively. Attaching this piece recursively to the diagram in Figure 2 gives a diagram for each $n \geq 41$ with $n \equiv 41(\bmod 12)$. Again the necessary information about $\langle\sigma, \tau\rangle$ containing a $p$-cycle, 


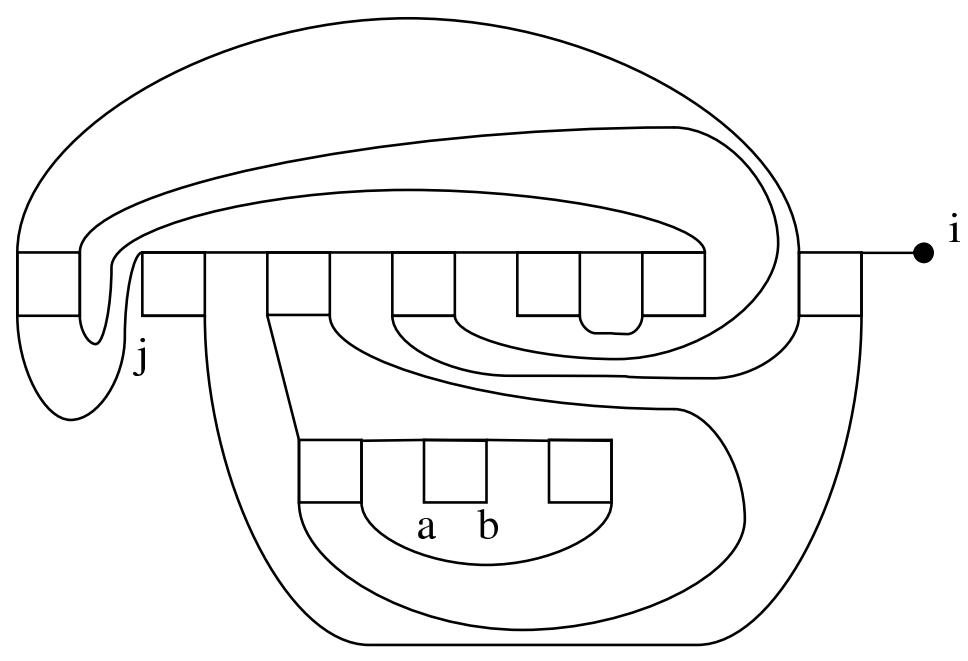

FiguRE 2. $\Sigma_{n}$ for $n \equiv 41(12)$ and $n \geq 41$

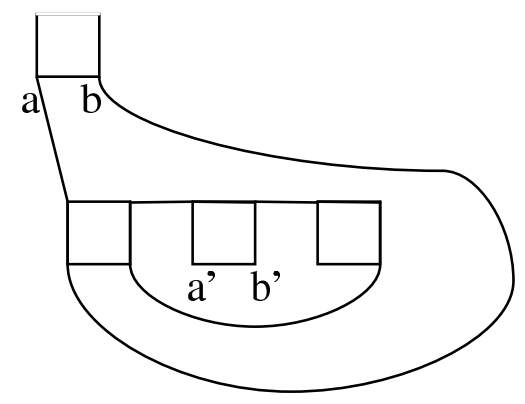

FIGURE 3. additional piece

which contains a letter and its image under $\sigma$ and a possibly different letter and its image under $\tau$, can be found in Table I. Each $n \geq 41$ with $n \equiv 41(\bmod 12)$ satisfies the conditions of at least one of the rows of Table I. The word in $\sigma$ and $\tau$ in that row then has some power which is a $p$-cycle for the prime $p$ in the last column. A similar recursive procedure on the diagrams for the groups $\Sigma_{n}$ with $42 \leq n \leq 52$ together with the information in Table I shows that the remaining groups $B_{n}$ with $n \geq 54$ are also $(2,4,6)$ groups. This completes the demonstration that every $B_{n}$ with $n \geq 41$ is a $(2,4,6)$ group and finishes the proof of the main theorem. 


\begin{tabular}{|c|c|c|c|c|}
\hline$n$ value & \multicolumn{2}{|c|}{ conditions on $m$} & word in $\sigma$ and $\tau$ & prime \\
\hline$n=41+12 m$ & $m \not \equiv 0(13)$ & & $\overline{\sigma \tau \sigma \tau \sigma}$ & 13 \\
\hline$n=41+12 m$ & $m \not \equiv 8(13)$ & $m \not \equiv 9(13)$ & $\sigma^{3} \tau \sigma \tau \sigma \tau \sigma^{2} \tau$ & 13 \\
\hline$n=42+12 m$ & $m \not \equiv 1(7)$ & & $\sigma \tau \sigma^{3} \tau$ & 7 \\
\hline$n=42+12 m$ & $m \not \equiv 2(7)$ & $m \not \equiv 4(7)$ & $\sigma^{3} \tau \sigma \tau \sigma^{2} \tau \sigma \tau \sigma^{2} \tau \sigma^{3} \tau \sigma^{2}$ & 7 \\
\hline$n=43+12 m$ & $m \not \equiv 4(7)$ & & $\tau \sigma \tau \sigma^{3}$ & 7 \\
\hline$n=43+12 m$ & $m \not \equiv 2(7)$ & $m \not \equiv 3(7)$ & $\tau \sigma^{2} \tau \sigma \tau \sigma \tau \sigma^{2} \tau \sigma \tau \sigma^{2} \tau \sigma \tau$ & 7 \\
\hline$n=44+12 m$ & $m \not \equiv 1(13)$ & & $\tau \sigma \tau \sigma^{3} \tau \sigma \tau \sigma^{3} \tau \sigma$ & 13 \\
\hline$n=44+12 m$ & $m \not \equiv 9(13)$ & $m \geq 1$ & $\sigma^{2} \tau \sigma^{2} \tau \sigma \tau \sigma^{3} \tau \sigma \tau \sigma \tau \sigma^{3}$ & 13 \\
\hline$n=45+12 m$ & $m \not \equiv 5(7)$ & $m \not \equiv 6(7)$ & $\sigma^{2} \tau \sigma^{2} \tau \sigma$ & 7 \\
\hline$n=45+12 m$ & $m \not \equiv 1(7)$ & $m \not \equiv 3(7)$ & $\tau \sigma^{3} \tau \sigma \tau \sigma \tau \sigma^{3} \tau \sigma^{2}$ & 7 \\
\hline$n=46+12 m$ & $m \not \equiv 0(7)$ & & $\tau \sigma^{3} \tau \sigma^{2} \tau \sigma \tau \sigma^{2} \tau \sigma^{3} \tau \sigma^{2} \tau \sigma \tau \sigma^{3} \tau \sigma \tau$ & 7 \\
\hline$n=46+12 m$ & $m \not \equiv 1(7)$ & $m \not \equiv 5(7)$ & $\tau \sigma^{2} \tau \sigma^{3} \tau \sigma \tau \sigma \tau \sigma^{3} \tau \sigma$ & 7 \\
\hline$n=47+12 m$ & $m \not \equiv 5(7)$ & $m \not \equiv 6(7)$ & $\sigma^{2} \tau \sigma \tau \sigma \tau \sigma^{3} \tau$ & 7 \\
\hline$n=47+12 m$ & $m \not \equiv 1(7)$ & $m \not \equiv 3(7)$ & $\sigma \tau \sigma^{2} \tau \sigma \tau \sigma \tau \sigma^{2} \tau \sigma^{3} \tau$ & 7 \\
\hline$n=48+12 m$ & $m \not \equiv 1(13)$ & & $\sigma \tau \sigma \tau \sigma$ & 13 \\
\hline$n=48+12 m$ & $m \not \equiv 11(13)$ & $m \geq 1$ & $\sigma \tau \sigma \tau \sigma^{2} \tau \sigma^{3} \tau$ & 13 \\
\hline$n=49+12 m$ & $m \not \equiv 5(7)$ & $m \not \equiv 6(7)$ & $\sigma^{2} \tau \sigma \tau \sigma \tau \sigma^{3} \tau$ & 7 \\
\hline$n=49+12 m$ & $m \not \equiv 1(7)$ & $m \not \equiv 3(7)$ & $\sigma^{2} \tau \sigma \tau \sigma^{3} \tau \sigma \tau \sigma^{3} \tau \sigma \tau \sigma^{2} \tau \sigma$ & 7 \\
\hline$n=50+12 m$ & $m \not \equiv 4(7)$ & & $\tau \sigma \tau \sigma \tau \sigma^{2} \tau \sigma^{2} \tau \sigma \tau \sigma \tau$ & 7 \\
\hline$n=50+12 m$ & $m \not \equiv 6(7)$ & $m \geq 2$ & $\sigma^{2} \tau \sigma \tau \sigma^{3} \tau \sigma \tau \sigma^{2} \tau \sigma^{3} \tau \sigma^{3}$ & 7 \\
\hline$n=51+12 m$ & $m \not \equiv 6(7)$ & & $\sigma^{3} \tau \sigma^{2} \tau \sigma \tau \sigma \tau \sigma^{2} \tau \sigma^{2} \tau$ & 7 \\
\hline$n=51+12 m$ & $m \not \equiv 3(7)$ & & $\sigma \tau \sigma^{2} \tau \sigma \tau \sigma^{2} \tau \sigma \tau \sigma^{3} \tau \sigma \tau \sigma^{3}$ & 7 \\
\hline$n=52+12 m$ & $m \not \equiv 6(11)$ & & $\tau \sigma^{2} \tau \sigma \tau \sigma^{2} \tau \sigma^{3}$ & 11 \\
\hline$n=52+12 m$ & $m \not \equiv 10(11)$ & $m \geq 1$ & $\sigma^{2} \tau \sigma^{2} \tau \sigma \tau \sigma \tau \sigma^{2}$ & 11 \\
\hline
\end{tabular}

TABLE I. Demonstration of $p$-cycles

\section{REFERENCES}

[1] S. A. Broughton. Classifying finite group actions on surfaces of low genus. J. Pure Appl. Algebra 69 (1991), 233-270.

[2] W. Burnside. Theory of groups of finite order (Cambridge University Press, 1911).

[3] M. D. E. Conder. Generators for alternating and symmetric groups. J. London Math. Soc. (2) 22 (1980), 75-86.

[4] M. D. E. Conder. More on generators for alternating and symmetric groups. Quart. J. Math. Oxford (2) 32 (1981), 137-163.

[5] M. D.E. Conder. Some results on quotients of triangle groups. Bull. Austral. Math. Soc. 29 (1984), 73-90.

[6] M.D.E. Conder, R.A. Wilson, and A.J. Woldar. The symmetric genus of sporadic groups. Proc. Amer. Math. Soc. 116 (1992), 653-663.

[7] B. Everitt. Permutation representations of the (2,4,r) Groups. Bull. Austral. Math. Soc. 49 (1994), 499-511. 
[8] The GAP Group, GAP — Groups, Algorithms, and Programming, Version 4.2; Aachen, St Andrews, 1999. (http://www-gap.dcs.st-and.ac.uk/ gap)

[9] H. H. Glover and D. Sjerve. The genus of $P S L_{2}(q)$. J. Reine Angew. Math. 380 (1987), 59-86.

[10] H. H. Glover and D. Sjerve. Representing $P S L_{2}(p)$ on a Reimann surface of least genus. Enseignment Math. 31 (1985), 305-325.

[11] J. L. Gross and T. W. Tucker. Topological graph theory (Wiley-Interscience, 1987).

$[12]$ C. L. May and J. Zimmerman. Groups of small strong symmetric genus. J. Group Theory 3 (2000), 233-245.

[13] V.S. Sikora. Two-element bases of hyperoctahedral groups. Visn. Kiiv. Univ. Ser. Fiz.-Mat. Nauki 1 (1999), 87-93.

[14] D. Singerman. Symmetries of Riemann surfaces with large automorphism group. Math. Ann. 210 (1974), 17-32.

[15] D. Singerman. Subgroups of Fuchsian groups and finite permutation groups. Bull. London Math. Soc. 2 (1970), 319-323.

[16] T. W. Tucker. Finite groups acting on surfaces and the genus of a group. J. Combin. Theory Ser. B 34 (1983), 82-98.

[17] VIGRE Working Group on Geometric Group Theory. Preprint. The Ohio State University (2000).

[18] S. N. Voon. The genus of $S L_{2}\left(F_{q}\right)$. Michigan Math. J. 40 (1993), 527-544.

[19] H. Wielandt. Finite permutation groups (Academic Press, 1964).

[20] A. White. Graphs, groups and surfaces, North-Holland Mathematical Studies. vol. 8 (NorthHolland, 1973).

[21] R. A. Wilson. The Monster is a Hurwitz group. J. Group Theory 4 (2001), 367-374.

[22] R. A. Wilson. The symmetric genus of the Fischer group $\mathrm{Fi}_{23}$. Topology 36 (1997), 379-380.

[23] R. A. Wilson. The symmetric genus of the Baby Monster. Quart. J. Math. Oxford Ser. (2) 44 (1993), 513-516. 